\title{
Prebiotic Matter in Space
}

\section{Pascale Ehrenfreund, Andreas Elsaesser and J. Groen}

Leiden Institute of Chemistry, P O Box 9502, 2300RA Leiden, The Netherlands
email: p.ehrenfreund@chem.leidenuniv.nl

\begin{abstract}
A significant number of molecules that are used in contemporary biochemistry on Earth are found in interstellar and circumstellar regions as well as solar system environments. In particular small solar system bodies hold clues to processes that formed our solar system. Comets, asteroids, and meteorite delivered extraterrestrial material during the heavy bombardment phase $\sim 3.9$ billion years ago to the young planets, a process that made carbonaceous material available to the early Earth. In-depth understanding of the organic reservoir in different space environments as well as data on the stability of organic and prebiotic material in solar system environments are vital to assess and quantify the extraterrestrial contribution of prebiotic sources available to the young Earth.
\end{abstract}

Keywords. Prebiotic matter, comets, asteroids, meteorites, carbonaceous material, early Earth

\section{The cycle of organic molecules in space}

Recent observations obtained from space missions and Earth-based telescopes have led to important discoveries in the field of molecular detection. The carbon chemistry seems to follow common pathways throughout the Universe. Interstellar dense molecular clouds and circumstellar envelopes are astrophysical environments providing the conditions for complex molecular synthesis (Ehrenfreund \& Charnley 2000, Kwok 2009, Herbst \& van Dishoeck 2009). But also our solar nebula was chemically active and dynamic as evidenced by results from the Stardust mission and recent theoretical calculations (Brownlee et al. 2006, Ciesla \& Sandford 2012). Currently 175 molecules are detected in the interstellar and circumstellar gas; 53 molecules are found in extragalactic sources (http://www.astro.uni-koeln.de). A lot of progress has been made to reveal the nature of the polycyclic aromatic hydrocarbon (PAHs) distribution, dust and carbonaceous grains in space. New results on the abundance and composition of organic molecules detected in the cometary coma and through laboratory analyses of meteorites have improved our knowledge on the fraction of material that reached the early Earth during the extended Late Heavy Bombardment LHB triggered by rapid migration of giant planets (Gomes et al. 2005, Bottke et al. 2012). This material may have provided raw material useful for protocell assembly on Earth (Chyba et al. 1992, Ehrenfreund et al. 2002).

Carbonaceous extract of meteorites have shown 66 extraterrestrial amino acids in addition to many other numerous organic compounds in the soluble phase although the major carbon component in meteorite samples is composed of a macromolecular organic fraction. Ultra high resolution molecular analysis of the solvent accessible organic fraction of Murchison shows high molecular diversity and many molecules still remain to be detected. The highest abundance of amino acids is currently measured in primitive SR meteorites found in Antarctica (Martins et al. 2007). Extraterrestrial nucleobases have been confirmed by isotopic measurements (Martins et al. 2008). The Murchison and Lonewolf Nunataks 94102 meteorites contained also a diverse suite of nucleobases, which included three unusual and terrestrially rare nucleobase analogs. Parallel experiments on the formation mechanism showed the formation of nucleobases in reactions of ammonium 
cyanide (Callahan et al. 2011). There is a consensus that prebiotic compounds such as amino acids and nucleobases are formed by aqueous alteration within their parent bodies and in the solar nebula. The abundance, distribution and ratio of amino acids (e.g. the $\beta$-Alanine/AIB ratio) shows a consistent dependence on aqueous alteration ruling out radiation processing (Glavin \& Dworkin 2009). In order to understand how this material could have been useful for the origin of life on Earth it is crucial to determine the stability of organic compounds under elevated radiation and temperature conditions. Amino acid destruction has been monitored by many different techniques and shows a degradation mechanism leading to decarboxylation. A slower destruction is observed when amino acids are embedded in ice (Gerakines et al. 2012). The consensus is that amino acids and nucleobases must be shielded by ice or minerals in order to survive harsh conditions (in space) and on the young Earth. Two decades of successful experiments on the International Space Station (ISS) and free-flying satellites have provided new information about the evolution of organic and biological material in space and planetary environments; some of them could not be reconciled with ground truth experiments (Guan et al. 2010, Mattioda et al. 2012).

Another important question is how prebiotic molecules can assemble on the early Earth? The prebiotic availability of fatty acids or their precursors and their properties of encapsulation, high permeability, and membrane growth, make them ideal model systems for investigating primitive forms of life. We have recently shown that PAH derivatives can be incorporated into fatty acids membranes and measured the first indication of a cholesterol-like stabilizing effect of oxidized PAH derivatives in a simulated prebiotic membrane (Groen et al. 2012).

In order to understand the link between extraterrestrial chemistry, the delivery of organic material to the young planets via small bodies and the origin of life on Earth it is crucial to investigate the inventory and the stability of organic material in different astronomical environments and on the early Earth. Contemporary biomolecules are not very stable against radiation or temperature cycles which invoke the presence of possible more stable precursor molecules like aromatic molecules (Ehrenfreund et al. 2006).

\section{References}

Bottke, W. F., et al. 2012, Nature 485/7396, 78-81

Brownlee, D., et al. 2006, Science 314, 1711-1716

Callahan, M. P., et al. 2011, PNAS 108(34), 13995-13998

Chyba, C. \& Sagan, C. 1992, Nature 355, 125-132

Chiesla, F. J. \& Sandford, S. A. 2012, Science 336/6080, 452

Ehrenfreund, P. \& Charnley, S. B. 2000, ARAA 38, 427-483

Ehrenfreund, P., et al. 2002, Reports on the Progress in Physics 65, 1427-1487

Ehrenfreund, P., Rasmussen, S., Cleaves, J. H., \& Chen, L., 2006, Astrobiology 6/3, 490-520

Gerakins, P. A., Hudson, R. L., Moore, M. H., \& Bell, L. 2012 Icarus 220, 647-659

Glavin, D. P. \& Dworkin, J. P. 2009, PNAS 106, 5487-5492

Gomes, R., Levison, H. F., Tsiganis, K., \& Morbidelli, A. 2005, Nature 435, 466-469

Groen, J., Deamer, A., Kros, A., \& Ehrenfreund, P. 2012, OLEB 42, 295-306

Guan, Y., et al. 2010, Planetary and Space Science 58, 1327-1346

Herbst, E. \& van Dishoeck, E. F. 2009, ARAA 47, 427-480

Kwok, S. 2009, Int. Journal of Astrobiology 8/3, 161-167

Martins, Z., et al. 2007, Meteoritics \&S Planetary Science 42/12, 2125-2136

Martins, Z., et al. 2008, Earth and Planetary Science Letters 270, 130-136

Mattioda, A., et al. 2012, Astrobiology 12, 841-853 